\title{
EDITORIAL
}

\section{A Challenge and Chance for Soil Ecology}

\author{
Shunyao Zhuang* \\ Institute of Soil Science, Chinese Academy of Sciences, Nanjing, 210008, China
}

\author{
ARTICLE INFO \\ Article history \\ Received: 26 September 2021 \\ Accepted: 28 September 2021 \\ Published Online: 28 September 2021
}

Due to the excessive use of mineral resources, more greenhouse effect gases have been emitted into the atmosphere. The global warming effect induced by human activity has been intensified much more than ever before. Accordingly, ecology systems are being suffered significant changes over the world. Soil is one of our most important natural resources. Physically, soil is the crust surface of the earth that can supply nutrients and support for plant growth. It often determines the nature of the vegetation present and related animals in number and types. Soils provide habitats for a myriad of living organisms, from small mammals and reptiles to tiny insects to microscopic cells of unimaginable numbers and diversity. Moreover, soil plays an important role as an engineering medium in human-built ecosystems ${ }^{[1]}$. Therefore, the understanding of soil and its response to the global warming is important for the harmony of human and environment. Generally, soil has four major components as air, water, mineral matter, and organic matter. The relative proportions of these four components greatly influence the behavior and productivity of soils. Through interactions of energy flow and mass exchange, soil plays the role as the crucial interface medium for air, minerals, water and life, and forms a complex integrated body, ecosystem. To a great extent, soil ecology is one new branch of ecology that study the relationship between soil biota and environment, including ecological structure, function, balance and evolution of soil ecosystem. The application of soil ecology will be a benefit for the reasonable and sustainable use of land resources and be important for agriculture, forestry and grazing production.

Soil ecology is concerned with interactions between organisms or between organisms and the soil environment. Soil ecology has its origins in soil biology and soil zoology, the study of organisms in the soil habitat. Soil ecology and soil science are related, yet different disciplines, with soil science focusing more on physical processes, the classification and genesis of soils, soil chemistry, and soil physics. Recent efforts in soil ecology have focused on developing a mechanistic understanding of how organisms and soils interact to yield patterns of soil biodiversity, nutrient cycling function within ecosystems, and feedbacks to global change mechanisms ${ }^{[2]}$. Many achievements have been made last several decades. However, there are still many challenges faced in research. Most studies are independent case studies

*Corresponding Author:

Shunyao Zhuang,

Institute of Soil Science, Chinese Academy of Sciences, Nanjing, 210008, China;

Email: syzhuang@issas.ac.cn 
based on either short-term field surveys or laboratory incubations. As a result, the findings are not systematic and adequate for generalization. Accordingly, it is important to establish more controlled field facilities or experiments and conduct more cross-site collaborative experiments ${ }^{[3]}$. Moreover, some critical scientific questions should be considered in the future research to face the challenges. Simultaneously, there is a great chance for researchers to contribute and push the study of this field ahead significantly. Research in Ecology, a peerreview and open access journal, provides a good service platform to publish these related study results. Certainly, all ecology-related topics could be accepted to publish on this journal.

\section{References}

[1] Brady NC, Weil RR. 2002. The nature and properties of soils. Prentice Hall, NJ, USA.

[2] Pavao-Zuckerman MA. 2008. Soil Ecology. General Ecology, pp.3279-3293.

[3] Semenov MV. 2021. Metabarcoding and metagenomics in soil ecology research: achievements, challenges, and prospects. Biology Bulletin Reviews 11, 40-53. 